\title{
Bridging the Mid-Infrared-to-Telecom Gap with Silicon Nanophotonic Spectral
}

\section{Translation}

\section{Xiaoping Liu ${ }^{1, \hbar, *}$, Bart Kuyken ${ }^{2, *}$, Gunther Roelkens ${ }^{2}$, Roel Baets ${ }^{2}$, Richard M. Osgood, Jr. ${ }^{1}$, and William M. J. Green ${ }^{3, \dagger}$}

Expanding far beyond traditional applications in optical interconnects at telecommunications wavelengths, the silicon nanophotonic integrated circuit platform has recently proven its merits for working with mid-infrared (mid-IR) optical signals in the 2-8 $\mu \mathrm{m}$ range. Silicon's broadband transparency $^{1,2}$, strong optical confinement, and potential for co-integration with CMOS electronics $^{3}$ are but a few of the many characteristics making the silicon platform ideal for development of high-performance, densely-integrated mid-IR optical systems. These systems are capable of addressing applications including industrial process and environmental monitoring ${ }^{4}$, threat detection ${ }^{5}$, medical diagnostics ${ }^{6}$, and free-space communication ${ }^{7}$. Rapid progress has led to the demonstration of various silicon components designed for the on-chip processing of mid-IR signals, including waveguides ${ }^{8-10}$, vertical grating couplers $^{11}$, microcavities $^{12,13}$, and electrooptic modulators ${ }^{14}$. Even so, a notable obstacle to the continued advancement of chip-scale systems is imposed by the narrow-bandgap semiconductors, such as InSb and $\mathrm{HgCdTe}$, traditionally used to convert mid-IR photons to electrical currents. The cryogenic or multi-stage thermo-electric cooling required to suppress dark current noise ${ }^{15}$, exponentially dependent upon the ratio $\mathrm{E}_{\mathrm{g}} / \mathrm{kT}$, can limit the development of small, low-power, and low-cost integrated optical systems for the mid-IR. However, if the mid-IR optical signal could be spectrally translated to shorter

\footnotetext{
${ }^{1}$ Department of Electrical Engineering, Columbia University, 1300 S. W. Mudd Building, 500 W. $120^{\text {th }}$ Street, New York, New York 10027, ${ }^{2}$ Photonics Research Group, Department of Information Technology, Ghent UniversityImec, Ghent B-9000, Belgium, ${ }^{3}$ IBM T. J. Watson Research Center, Yorktown Heights, NY 10598, USA, †Current address: OFS Labs, 19 Schoolhouse Road, Somerset, NJ 08873, USA. *These authors contributed equally to this work. ${ }^{\dagger}$ wgreen@us.ibm.com
} 
wavelengths, for example within the near-infrared telecom band, photodetectors using wider bandgap semiconductors such as InGaAs or Ge could be used to eliminate prohibitive cooling requirements. Moreover, telecom band detectors typically perform with higher detectivity and faster response times when compared with their mid-IR counterparts ${ }^{15}$. Spectral translators employing sum or difference frequency generation in nonlinear crystals, including $\mathrm{LiNbO}_{3}{ }^{16-18}$ and $\mathrm{KTP}^{19}$, have been studied. However, such systems can be impeded by low conversion efficiencies, their significant size, and limited integrability of their component parts. Here we address these challenges with a silicon-integrated approach to spectral translation, by employing efficient four-wave mixing (FWM) and large optical parametric gain in silicon nanophotonic wires $^{20-22}$. Using an optical pump near silicon's two-photon absorption (TPA) threshold ${ }^{23}$, we excite nanophotonic wires uniquely engineered to use higher-order waveguide dispersion to facilitate spectral translation of a mid-IR input signal at $2440 \mathrm{~nm}$ to the telecom band at $1620 \mathrm{~nm}$, across a span of $62 \mathrm{THz}$. The converted signal simultaneously experiences a translation gain of more than $19 \mathrm{~dB}$, an efficiency enhancement which can dramatically improve the detection sensitivity for weak mid-IR signals. Moreover, this single silicon device also performs as a transmitter, by converting telecom band signals to the mid-IR with a translation gain of $8.0 \mathrm{~dB}$. Finally, an $8.4 \mathrm{~dB}$ optical parametric amplification of telecom band signals is demonstrated when using a mid-IR pump, reinforcing the wide-ranging technological role silicon nanophotonic wires can serve within both the mid-IR and telecom bands.

Mid-IR to telecom band spectral translation in silicon wires can be accomplished using efficient FWM with discrete band phase-matching ${ }^{24,25}$. In this process, the pump is placed away from the zero dispersion wavelength, and higher-order waveguide dispersion is used to phase- 
match a discrete pair of bands at spectrally distant frequencies, located symmetrically on either side of the pump. Discrete band phase-matching can be achieved in a waveguide with anomalous $2^{\text {nd }}$-order dispersion $\left(\beta_{2}<0\right)$ and small positive $4^{\text {th }}$-order dispersion $\left(\beta_{4}>0\right)$ (see Supplementary Figure 1), conditions which are engineered ${ }^{21}$ through manipulating the crosssectional dimensions and cladding materials of the silicon nanophotonic wire. Figure 1a shows an optical microscope image of the $2 \mathrm{~cm}$ long silicon nanophotonic wire used here for spectral translation, as fabricated on a $200 \mathrm{~mm}$ silicon-on-insulator (SOI) wafer at imec, through the multi-project-wafer service ePIXfab (www.ePIXfab.eu). The entire length of the wire is coiled into a compact spiral, occupying an on-chip footprint of only $625 \mu \mathrm{m}$ by $340 \mu \mathrm{m}$. The wire has cross-sectional dimensions of $w=900 \mathrm{~nm}$ by $h=220 \mathrm{~nm}$, as shown in Fig. $1 \mathrm{~b}$. The cladding consists of air above and a $2 \mu \mathrm{m}$ buried oxide $\left(\mathrm{SiO}_{2}\right)$ below the silicon core. Numerical simulations indicate that the dispersion conditions $\beta_{2}<0$ and $\beta_{4}>0$ are satisfied over the spectral range from 1810-2410 nm (see Supplementary Figure 2). Over a similar range, a large effective nonlinearity parameter of $\gamma \sim 130(\mathrm{~W} \cdot \mathrm{m})^{-1}$ and a low propagation loss of $2.6 \mathrm{~dB} / \mathrm{cm}$ also serve to facilitate highly efficient FWM for this compact device.

The nonlinear mixing and spectral translation characteristics of the silicon nanophotonic wire are illustrated in Figs. 1c-d. Figure 1c shows the recorded output spectrum when the wire is excited by a pump pulse-train at $1946 \mathrm{~nm}$ having a peak coupled input power of $37.3 \mathrm{~W}$ (for experimental details see Methods). While this pump wavelength is not yet beyond silicon's TPA threshold of $2200 \mathrm{~nm}^{23}$, the TPA coefficient is nevertheless a factor of 2-3x lower than that at $1550 \mathrm{~nm}$, resulting in small nonlinear loss and efficient FWM. For example, in the absence of any probe signal (Signal OFF), the pump transmission spectrum already exhibits clear signatures 
of the desired phase-matched FWM processes. Specifically, strong broadband modulation instability (MI) peaks ${ }^{22}$ appear adjacent to the pump at $1810 \mathrm{~nm}$ and $2090 \mathrm{~nm}$. Moreover, two additional discrete MI bands with much larger detuning from the pump appear at $1620 \mathrm{~nm}$ and $2440 \mathrm{~nm}$, and serve as direct evidence of higher-order phase-matching. The absolute power of the MI band at $2440 \mathrm{~nm}$ appears lower than that of the MI band at $1620 \mathrm{~nm}$, due to a $1.8 \mathrm{~dB}$ asymmetry expected from the Manley-Rowe power division relations, as well as from $\sim 3-4 \mathrm{~dB}$ larger losses in the output fibre optical collection path at longer wavelengths.

The visibility of the MI bands, associated with the parametric amplification of background noise, suggests that the pumped silicon nanophotonic wire should exhibit significant parametric gain as well as a large conversion efficiency when probed by input signals at these wavelengths. Figure $1 \mathrm{~d}$ illustrates the output spectrum in one such case, when the long-wavelength discrete MI band is probed (Signal ON) by a low-power $\left(\mathrm{P}_{\text {sig }}<35 \mu \mathrm{W}\right)$ continuous-wave mid-IR signal at $2440 \mathrm{~nm}$. When the signal is tuned into resonance with this spectral band, it experiences strong parametric amplification through degenerate FWM (evidenced by the appearance of the large spectral pedestal), and is simultaneously up-converted to a prominent telecom band idler at 1620 nm. This large spectral translation over more than $62 \mathrm{THz}$ illustrates that the higher-order dispersion design methodology applied here may be used to efficiently convert optical information on a mid-IR carrier into the telecom band, where it can be detected and processed using un-cooled, high-speed, high-sensitivity III-V and group-IV semiconductor detector technologies. 
By recording transmission spectra for a range of signal wavelengths near $2440 \mathrm{~nm}$ and 1620 $\mathrm{nm}$, the wavelength dependence of spectral translation efficiency and parametric gain within the discrete phase-matching bands can be determined (see Methods). Figure 2a illustrates that for mid-IR input signals, the silicon wire device attains optical transparency (on-chip gain exceeding $0 \mathrm{~dB}$ ) across a bandwidth of $150 \mathrm{~nm}$ near the signal and $45 \mathrm{~nm}$ near the idler. The data also demonstrates that spectral translation of mid-IR signals to the telecom band idler near $1620 \mathrm{~nm}$ takes place with a peak conversion gain of $19.5 \mathrm{~dB}$. Therefore, not only could such a spectral translator facilitate detection of mid-IR signals without cumbersome cooling requirements, the associated optical gain could also dramatically improve the sensitivity of such a receiver system, particularly for weak mid-IR input levels ${ }^{26}$. At the same time, the mid-IR input signal experiences a peak on-chip parametric amplification of $18.8 \mathrm{~dB}$.

Figure $2 \mathrm{~b}$ shows the result of a similar set of experiments, in which a telecom band input signal is tuned across the MI peak near $1620 \mathrm{~nm}$. In this case, the on-chip transparency bandwidth is approximately $20 \mathrm{~nm}$ for the signal and $40 \mathrm{~nm}$ for the idler. The telecom band signal is spectrally translated to the mid-IR with a peak gain of $8.0 \mathrm{~dB}$, a process which can be applied to generating and transmitting arbitrary mid-IR signals using commercially-available telecom components ${ }^{27,28}$. In addition to performing the spectral translation function, Fig. $2 b$ illustrates that the telecom band input signal is simultaneously amplified by $8.4 \mathrm{~dB}$. The demonstration of a silicon wire amplifier which utilizes a mid-IR pump to provide substantial parametric gain to a telecom band signal is of particular technological significance, as such an amplifier could find applications within the CMOS-integrated silicon nanophotonic platforms currently being developed for high-speed optical interconnect systems ${ }^{3}$. 
The spectral translation of the telecom band signal to the mid-IR shown in Fig. $2 \mathrm{~b}$ occurs with an approximately $11 \mathrm{~dB}$ gain reduction when compared with the reversed scenario illustrated in Fig. 2a. As the energetic combination of a $1620 \mathrm{~nm}$ signal photon with a pump photon lies significantly above silicon's bandgap, the observed asymmetry is expected due to the effects of non-degenerate $\mathrm{TPA}^{23,24}$ in the silicon wire. Non-degenerate TPA produces larger attenuation of an input signal near $1620 \mathrm{~nm}$ as compared to one near $2440 \mathrm{~nm}$, when each is combined with the strong pump at $1946 \mathrm{~nm}$. Therefore, larger gain values could be expected for the telecom band signal if the pump wavelength were increased.

The phase-matched signal and idler wavelengths linked by the discrete FWM spectral translation process are dependent upon the magnitudes of $\beta_{2}$ and $\beta_{4}$, and can therefore be tuned by selection of the wavelength at which the dispersive silicon nanophotonic wire is pumped. The spectral separation between bands is expected to increase for large values of $\left|\beta_{2}\right|$ and small values of $\left|\beta_{4}\right|$. Figure 3a plots the spectral locations at which the discrete signal and idler MI bands appear as a function of the pump wavelength. The figure illustrates that the bands are maximally separated by $865 \mathrm{~nm}$ when the wire is pumped at $1998 \mathrm{~nm}$. Conversely, the bands are observed to move closer together as the pump approaches the two zero-GVD points located at $1810 \mathrm{~nm}$ and $2410 \mathrm{~nm}$.

An optimization of the silicon nanophotonic wire dispersion design, focusing upon increasing $\left|\beta_{2}\right|$ while simultaneously decreasing $\left|\beta_{4}\right|$, can facilitate translation of even longer wavelength mid-IR signals into the telecom band. For example, Fig. $3 \mathrm{~b}$ plots numerical calculations of the 
phase-matched discrete signal and idler wavelengths of a design tailored for translating a range of mid-IR signals from 3000-3550 nm into the L-band (see Methods). This spectrum is targeted for its overlap with a mid-IR low-loss window in $\mathrm{SiO}_{2}{ }^{1}$. This design consists of a silicon wire with a thickness of $300 \mathrm{~nm}$ and a width in the range of 700-900 nm, completely surrounded by an oxide cladding (see Supplementary Figure 4). In the specific case of a wire with $w=900 \mathrm{~nm}$ and $h=300 \mathrm{~nm}$, Fig. $3 \mathrm{~b}$ illustrates that an input signal at $3550 \mathrm{~nm}$ could be spectrally translated to an L-band idler at $1590 \mathrm{~nm}$ (and vice versa), using a pump wavelength of $2200 \mathrm{~nm}$. This corresponds to a span of $104 \mathrm{THz}$, more than an octave in optical frequency.

Moreover, while a pulsed pump with a high peak power is used in the present demonstration, a practical spectral translation system will require a continuous-wave (c.w.) pump, in order to eliminate any requirement for synchronization of the input mid-IR signals to the pump repetition period. Because the oxide-clad wire's dispersion characteristics were designed with the intent of operating the pump at or beyond silicon's TPA threshold at $2200 \mathrm{~nm}$, c.w. parametric gain is made possible by avoiding the deleterious effects of nonlinear loss and TPA-induced free-carrier absorption. Detailed calculations described in the Supplementary Methods and plotted in Supplementary Figure 5 show that c.w. spectral translation of a $3550 \mathrm{~nm}$ input signal to $1590 \mathrm{~nm}$ can be accompanied by a translation gain as large as $22 \mathrm{~dB}$, for a moderate pump power of 300 $\mathrm{mW}$ at $2200 \mathrm{~nm}$.

In conclusion, we have shown that judicious engineering of FWM processes in silicon nanophotonic wires can facilitate amplified bi-directional spectral translation of signals between the mid-IR and the telecom band, across a $62 \mathrm{THz}$ span in optical frequency. Telecom band 
detection of translated mid-IR signals can eliminate the burdensome cooling requirements traditionally associated with mid-IR photodetectors, and can be performed by on-chip photodetectors integrated via heterogeneous ${ }^{29}$ or monolithic ${ }^{30}$ techniques. Moreover, these spectral translation devices can be integrated with additional mid-IR and/or telecom band silicon nanophotonic components such as modulators, wavelength (de-)multiplexers, and switches, which together have the potential to produce flexible, chip-scale optical systems for mid-IR applications. 


\section{Methods:}

\section{Four-wave mixing experimental configuration:}

In our experiments, the FWM pump is a picosecond pulse train (FWHM $\sim 2 \mathrm{ps,} \mathrm{repetition}$ rate $=76 \mathrm{MHz}$ ) from a tunable optical parametric oscillator (mode-locked Ti:sapphire-pumped Coherent Mira-OPO). The pump spectrum has a signal-to-noise ratio larger than $70 \mathrm{~dB}$ over the wavelength range from $1600 \mathrm{~nm}$ to $2500 \mathrm{~nm}$ (see Supplementary Figure 3). The c.w. probe signals are obtained either from a tunable mid-IR laser (IPG Photonics SFTL $\mathrm{Cr}^{2+}: \mathrm{ZnSe}$ polycrystal with erbium-fibre laser pump source) or a tunable telecom laser (Agilent 81640A). Pump and probe are coupled into two separate single-mode optical fibres, aligned individually with polarization controllers to excite the fundamental quasi-TE mode in the silicon nanophotonic wire, and then multiplexed with a 90/10 fused fibre directional coupler. Coupling into/out of the $2 \mathrm{~cm}$-long silicon nanophotonic wire is accomplished via cleaved facet edge coupling with lensed tapered fibres (coupling losses $=10+/-1 \mathrm{~dB} /$ facet, across the entire spectral range utilized). The spectral content of the transmitted light is analyzed with $1 \mathrm{~nm}$ spectral resolution, using a mid-IR optical spectrum analyzer (Yokogawa AQ6375).

\section{Extraction of spectral translation efficiency and signal gain:}

The peak power of the spectrally translated idler pulse at the output of the silicon nanophotonic wire, $P_{\text {idler_out }}$, is derived from the measured FWM spectra, according to $P_{\text {idler_out }}=F\left(\int P_{\text {idler_avg }}(\lambda) d \lambda\right)$. In order to convert the time-averaged idler power $P_{\text {idler_avg }}$ measured by the OSA into peak power, the spectrally integrated power is weighted by the duty 
cycle factor $F=1 /(76 \mathrm{MHz} \cdot 2 \mathrm{ps})$ to account for the pulsed nature of the experiment. A similar procedure is applied to calculate the signal output power $P_{\text {signal_out }}$. A $2 \mathrm{~nm}$ wide band-stop filter is first numerically applied to the time-averaged signal spectrum, in order to reject the power remaining in the narrowband c.w. tone. The peak signal power is then computed according to $P_{\text {signal_out }}=F\left(\int P_{\text {signal_filtered_avg }}(\lambda) d \lambda\right)$. Finally, to find the c.w. signal power at the waveguide input $P_{\text {signal_in }}$, the output c.w. signal power is measured (with the pump off) and corrected to account for total propagation losses of $\alpha \mathrm{dB}$ incurred through the $2 \mathrm{~cm}$ long device, $P_{\text {signal_in }}=10^{\alpha / 10}\left(\int P_{\text {signal_out_pump_off }}(\lambda) d \lambda\right)$. The on-chip idler spectral translation gain $\eta$ is then defined as the ratio of peak idler power and input c.w. signal power, $\eta=P_{\text {idler_out }} / P_{\text {signal_in }}$. Accordingly, the on-chip parametric signal gain is given by $G=P_{\text {signal_out }} / P_{\text {signal_in }}$. The error bars in the on-chip parametric gain data are calculated to reflect the uncertainty in the total propagation loss $\alpha$, as well as the contribution of the MI noise background accumulated when integrating the signal/idler power at the waveguide output. Additional detail on the estimation of error bars is included in the Supplementary Methods.

\section{Numerical calculations of signal and idler discrete band tuning versus pump wavelength:}

The linear phase-mismatch $\Delta k_{l}$ is characterized by $\Delta k_{l}=\beta_{s}+\beta_{i}-2 \beta_{p}$, where $\beta_{s}, \beta_{i}$, and $\beta_{p}$ are the modal propagation constants for signal, idler, and pump respectively. These propagation constants and their spectral dispersion are obtained through numerical simulations, using a commercial finite-element eigen-mode solver (RSoft FemSim). Solving the phase-matching equation $\Delta k_{l}+2 \gamma P=0(P=300 \mathrm{~mW}$ is assumed for the calculation in Fig. 3b) with signal and idler frequencies constrained by the pump detuning $\Delta \omega=\left|\omega_{p}-\omega_{s}\right|=\left|\omega_{p}-\omega_{i}\right|$ will generally yield 
two solutions for $\Delta \omega$. The smaller-valued solution corresponds to broadband phase-matched signal-idler regions appearing immediately adjacent to the pump, and is ignored for the purposes of spectral translation in this work. The larger-valued of the two solutions describes the discrete signal and idler bands appearing at large pump detuning. The curves of phase-matched signalidler pairs shown in Fig. $3 b$ are found by repeating this calculation for a range of pump frequencies, and converting to detuning in units of wavelength. 


\section{References}

1. Soref, R. A., Emelett, S. J. \& Buchwald, W. R. Silicon waveguided components for the long-wave infrared region. Journal of Optics A: Pure and Applied Optics 8, 840-848 (2006).

2. Jalali, B. et al. Prospects for silicon mid-IR Raman lasers. IEEE Journal of Selected Topics in Quantum Electronics 12, 1618-1627 (2006).

3. Green, W. M. J. et al. in SEMICON (Chiba, Japan, 2010).

4. Willer, U., Saraji, M., Khorsandi, A., Geiser, P. \& Schade, W. Near- and mid-infrared laser monitoring of industrial processes, environment and security applications. Optics and Lasers in Engineering 44, 699-710 (2006).

5. Moore, D. S. Instrumentation for trace detection of high explosives. Review of Scientific Instruments 75, 2499-2512 (2004).

6. Namjou, K., Roller, C. B. \& McCann, P. J. in IEEE Circuits \& Devices Magazine 22-28 (2006).

7. Capasso, F. et al. Quantum cascade lasers: Ultrahigh-speed operation, optical wireless communication, narrow linewidth, and far-infrared emission. IEEE Journal of Quantum Electronics 38, 511-532 (2002).

8. Baehr-Jones, T. et al. Silicon-on-sapphire integrated waveguides for the mid-infrared. Optics Express 18, 12127-12135 (2010).

9. Mashanovich, G. Z. et al. Low loss silicon waveguides for the mid-infrared. Optics Express 19, 7112-7119 (2011). 
10. Li, F. et al. Low propagation loss silicon-on-sapphire waveguides for the mid-infrared. Optics Express 19, 15212-15220 (2011).

11. Kuyken, B. et al. in Integrated Photonics Research IMB6 (Ottawa, Canada, 2011).

12. Spott, A., Liu, Y., Baehr-Jones, T., Ilic, R. \& Hochberg, M. Silicon waveguides and ring resonators at 5.5 $\mu \mathrm{m}$. Applied Physics Letters 97, 213501 (2010).

13. Shankar, R., Leijssen, R., Bulu, I. \& Loncar, M. Mid-infrared photonic crystal cavities in silicon. Optics Express 19, 5579-5586 (2011).

14. Van Camp, M. A. et al. in Conference on Lasers and Electro-Optics CTu3I.2 (San Jose, USA, 2012).

15. Sze, S. M. Physics of Semiconductor Devices (John Wiley \& Sons, 1981).

16. Midwinter, J. E. \& Warner, J. Up-conversion of near infrared to visible radiation in lithium-meta-niobate. Journal of Applied Physics 38, 519-523 (1967).

17. Buchter, K. D. F., Herrmann, H., Langrock, C., Fejer, M. M. \& Sohler, W. All-optical Ti:PPLN wavelength conversion modules for free-space optical transmission links in the mid-infrared. Optics Letters 34, 470-472 (2009).

18. Temporao, G. et al. Mid-infrared single-photon counting. Optics Letters 31, 1094-1096 (2006).

19. Dam, J. S., Pedersen, C. \& Tidemand-Lichtenberg, P. High-resolution two-dimensional image upconversion of incoherent light. Optics Letters 35, 3796-3798 (2010).

20. Zlatanovic, S. et al. Mid-infrared wavelength conversion in silicon waveguides using ultracompact telecom-band-derived pump source. Nature Photonics 4, 561-564 (2010). 
21. Liu, X., Osgood, J., R. M., Vlasov, Y. A. \& Green, W. M. J. Mid-infrared optical parametric amplifier using silicon nanophotonic waveguides. Nature Photonics 4, 557$560(2010)$.

22. Kuyken, B. et al. $50 \mathrm{~dB}$ parametric on-chip gain in silicon photonic wires. Optics Letters 36, 4401-4403 (2011).

23. Bristow, A. D., Rotenberg, N. \& van Driel, H. M. Two-photon absorption and Kerr coefficients of silicon for 850-2200 nm. Applied Physics Letters 90, 191104 (2007).

24. Lin, Q., Johnson, T. J., Perahia, R., Michael, C. P. \& Painter, O. J. A proposal for highly tunable optical parametric oscillation in silicon micro-resonators. Optics Express 16, 10596-10610 (2008).

25. Tien, E. K. et al. Discrete parametric band conversion in silicon for mid-infrared applications. Optics Express 18, 21981-21989 (2010).

26. Huang, Y. et al. Electrical signal-to-noise ratio improvement in indirect detection of midIR signals by wavelength conversion in silicon-on-sapphire waveguides. Applied Physics Letters 99, 181122 (2011).

27. Zlatanovic, S. et al. Mid-infrared wavelength conversion in silicon waveguides pumped by silica-fiber-based source. IEEE Journal of Selected Topics in Quantum Electronics 18, $612-620(2012)$.

28. Ophir, N. et al. First demonstration of a 10-Gb/s RZ end-to-end four-wave-mixing based link at $1884 \mathrm{~nm}$ using silicon nanowaveguides. IEEE Photonics Technology Letters 24, 276-278 (2012).

29. Roelkens, G. et al. Integration of InP/InGaAsP photodetectors onto silicon-on-insulator waveguide circuits. Optics Express 13, 10102-10108 (2005). 
30. Assefa, S. et al. CMOS-integrated high-speed MSM germanium waveguide photodetector. Optics Express 18, 4986-4999 (2010). 


\section{End Notes:}

\section{Acknowledgements}

The authors gratefully acknowledge the staff at imec (Leuven, Belgium) where the silicon nanophotonic waveguide devices were fabricated. They would also like to thank Y. A. Vlasov (IBM Research) for many helpful and motivating discussions. B.K. acknowledges the Flemish Research Foundation (FWO) for a doctoral fellowship. This work was partially funded under the Methusalem "Smart Photonic Chips," FP7-ERC-INSPECTRA, and FP7-ERC-MIRACLE programs.

\section{Author contributions}

X.L. (x12165@columbia.edu) and B.K. (bart.kuyken@intec.ugent.be) performed the numerical dispersion and phase-matching design calculations. B.K., G.R., and R.B. supervised the waveguide device fabrication process. X.L. and B.K. performed the wavelength translation experiments with guidance and supervision from W.M.J.G. All authors contributed to the data analysis and writing of the manuscript.

\section{Author information}

The authors declare no competing financial interests. Correspondence and requests for materials should be addressed to W.M.J.G. (wgreen@us.ibm.com). 
Figure legends:
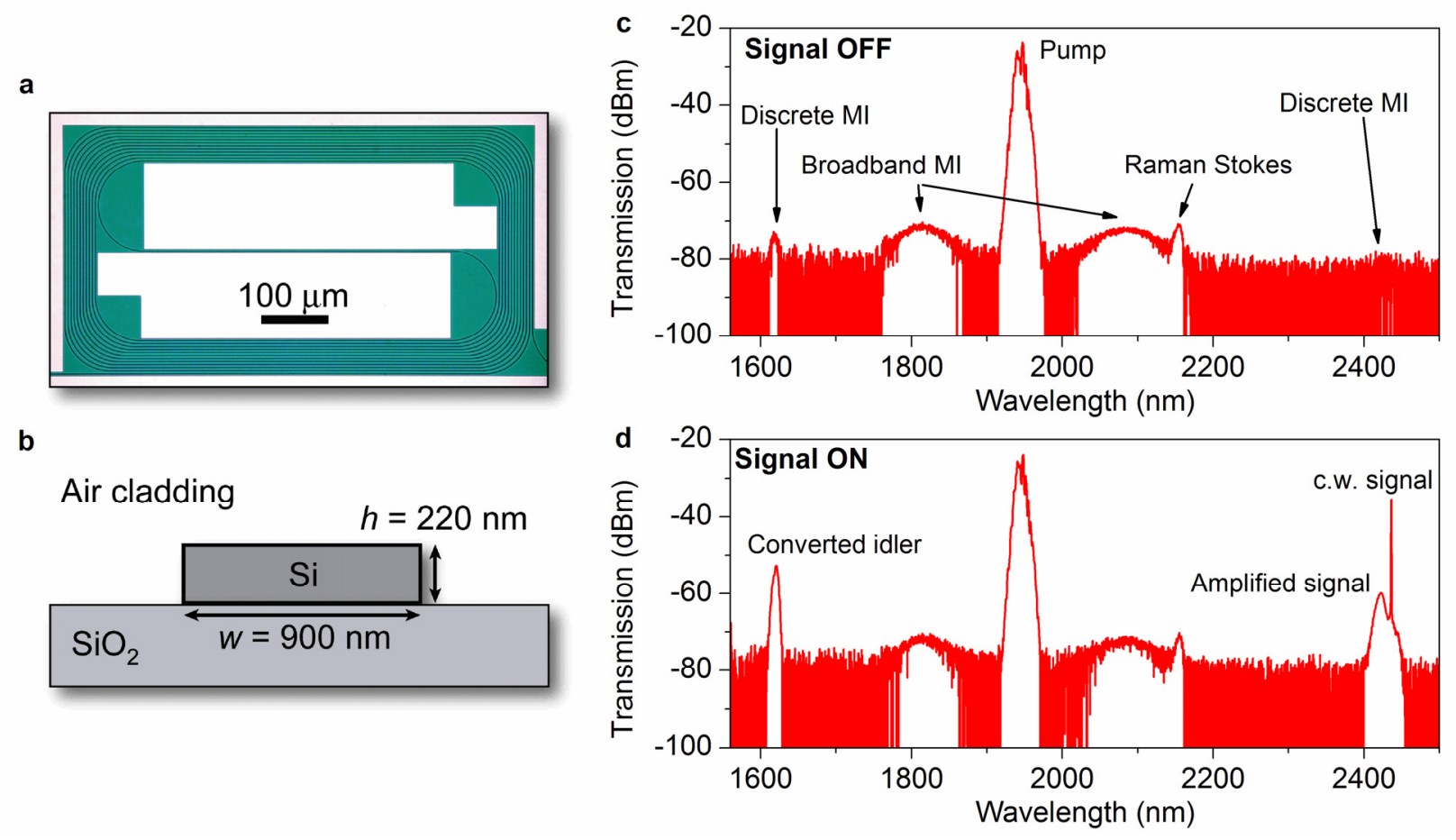

Figure 1 | Structural design and transmission characteristics of the silicon nanophotonic wire spectral translation device. a, Optical microscope image of the spiral-coiled silicon wire. The wire has a total length of $2 \mathrm{~cm}$, and occupies an on-chip footprint of only $625 \mu \mathrm{m} \times 340 \mu \mathrm{m}$. Bends with a conservative $60 \mu \mathrm{m}$ radius are used. b, Cross-sectional schematic, illustrating a silicon core with a width of $w=900 \mathrm{~nm}$ and a height of $h=220 \mathrm{~nm}$, which lies upon a $2 \mu \mathrm{m}$ thick $\mathrm{SiO}_{2}$ buried oxide layer. The silicon core is air-clad from above. c, Output transmission spectrum with pump operating at $\lambda=1946 \mathrm{~nm}$ and input signal OFF. The observed modulation instability spectrum generated by amplification of background noise serves as a marker of the spectral bands in which phase-matching conditions are met. The location of the broadband MI peaks adjacent to the pump at $1810 \mathrm{~nm}$ and $2090 \mathrm{~nm}$ is primarily determined by $\beta_{2}$, while the discrete MI bands at $1620 \mathrm{~nm}$ and $2440 \mathrm{~nm}$ occur as a result of higher-order phase-matching 
dictated by the values of both $\beta_{2}$ and $\beta_{4}$. A Raman Stokes peak is also observed at $2155 \mathrm{~nm}$. d, Transmission spectrum with input signal ON. A c.w. mid-IR signal is tuned to coincide with the discrete MI band at $2440 \mathrm{~nm}$. Parametric amplification of the signal occurs with simultaneous spectral translation across $62 \mathrm{THz}$, to an idler at $1620 \mathrm{~nm}$. 

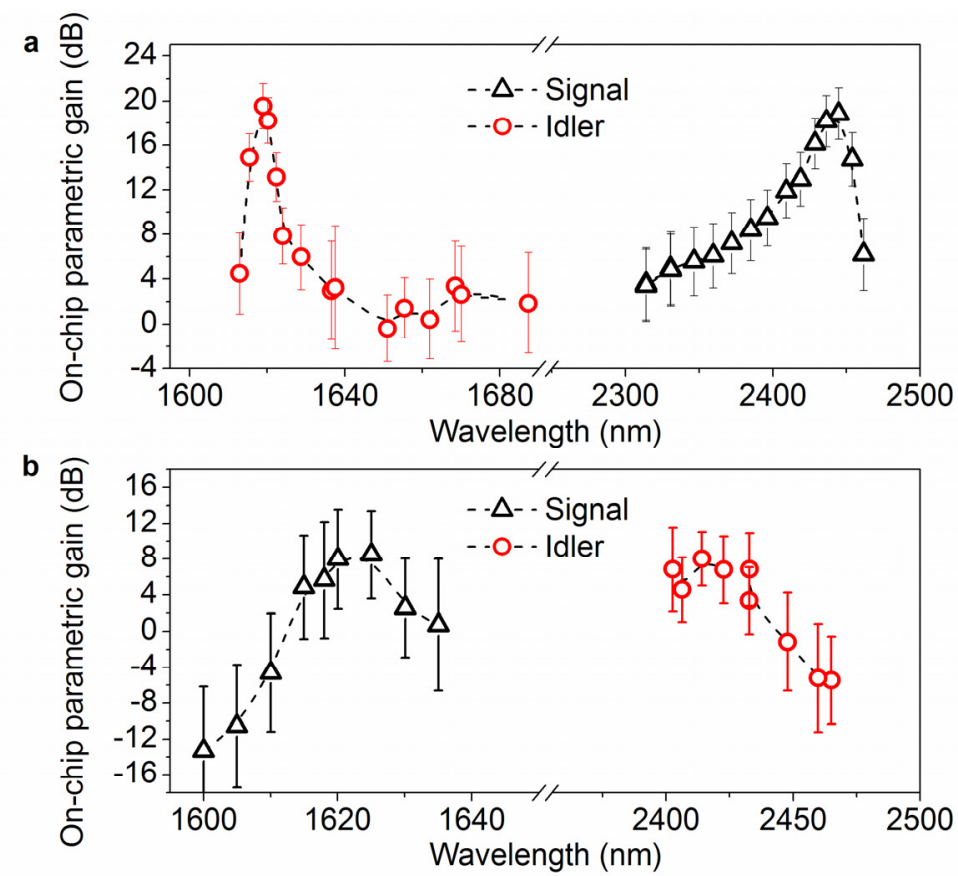

Figure 2 | Wavelength-resolved on-chip spectral translation efficiency and parametric signal gain. a, Injection of a mid-IR input signal $\left(\mathrm{P}_{\text {sig }}<35 \mu \mathrm{W}\right)$, with translation to a telecom band output idler. The peak on-chip translation efficiency is $19.5 \mathrm{~dB}$, while the signal gain is $18.8 \mathrm{~dB}$. The transparency bandwidth exceeds $45 \mathrm{~nm}$ near the idler, and $150 \mathrm{~nm}$ near the signal. b, Reversed scenario, with injection of a telecom band input signal $\left(\mathrm{P}_{\text {sig }}<50 \mu \mathrm{W}\right)$ and translation to a mid-IR output idler. The peak on-chip translation efficiency is $8.0 \mathrm{~dB}$, and the signal gain is $8.4 \mathrm{~dB}$. Transparency is reached over a bandwidth of $40 \mathrm{~nm}$ near the idler and $20 \mathrm{~nm}$ near the signal. In all of the above measurements, the silicon nanophotonic wire is pumped at $1946 \mathrm{~nm}$ with a peak power of $37.3 \mathrm{~W}$. The small shift in the spectral position of the mid-IR gain peak between Fig. 2a and Fig. 2b (2440 nm versus $2420 \mathrm{~nm}$, respectively) occurs as a result of pump wavelength drift. The dashed curves are included as a guide to the eye. Estimation of error bars is described in Supplementary Methods. 

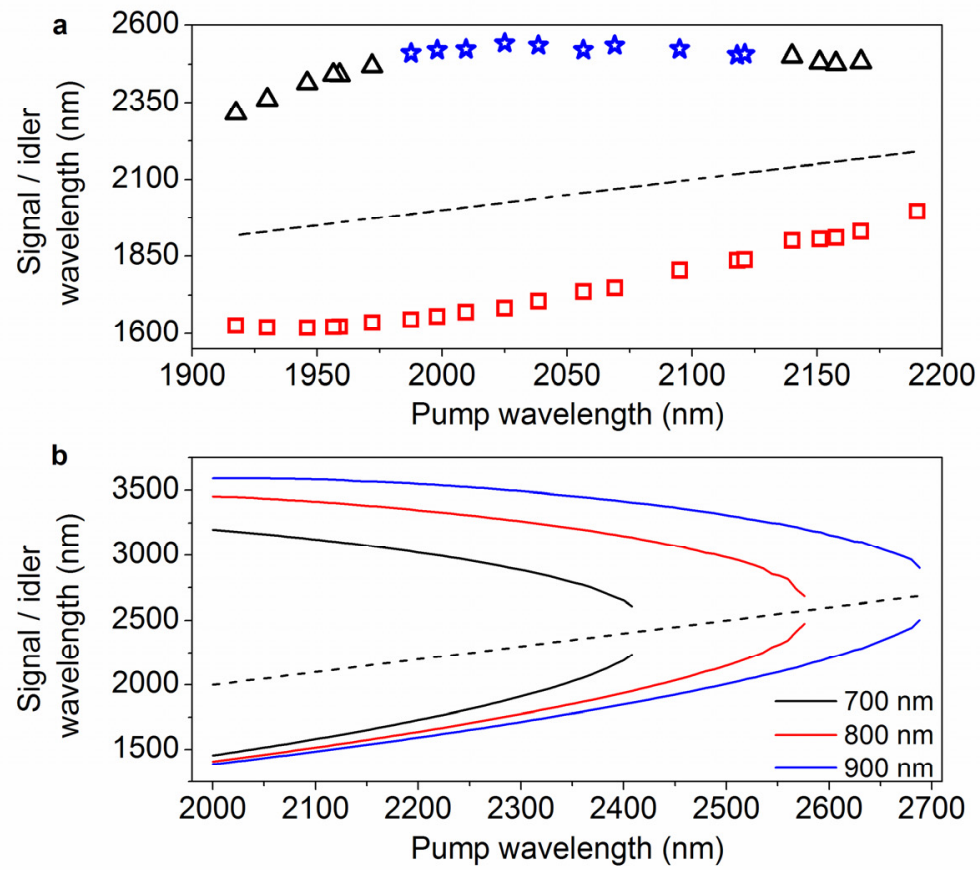

Figure 3 | Phase-matched signal and idler wavelengths linked by the silicon nanophotonic spectral translation process. a, The symbols mark the spectral locations of the discrete MI bands as a function of pump wavelength, for the experimentally demonstrated silicon wire having $w=900 \mathrm{~nm}, h=220 \mathrm{~nm}$. The MI bands indicated by blue stars were not measured directly, as they were located beyond the $2500 \mathrm{~nm}$ maximum wavelength limit of the spectrum analyzer used. The positions of these bands were therefore inferred from energy conservation. $\mathbf{b}$, Design calculations describing the phase-matched discrete band locations versus pump wavelength, for the fundamental quasi-TE mode of an $\mathrm{SiO}_{2}$-clad silicon wire with $h=300 \mathrm{~nm}$ and widths $w=700 \mathrm{~nm}, 800 \mathrm{~nm}$, and $900 \mathrm{~nm}$. The wires are tailored for spectral translation across more than an octave in optical frequency, between the 3000-3550 nm mid-IR range and the L-band. The calculations assume c.w. pumping with $300 \mathrm{~mW}$ pump power. In both panels, the dashed line marks the pump wavelength. 